\title{
ESCALA DE SATISFACCIÓN CON EL TRATAMIENTO RECIBIDO (CRES-4): LA VERSIÓN EN ESPAÑOL
}

Feixas, G. ${ }^{1}{ }^{2}$; Pucurull, O. ${ }^{1}$; Roca, C. ${ }^{1}$; Paz, C. ${ }^{1}$; García-Grau, E. ${ }^{1}$ y Bados, A. ${ }^{1}$

1 Universitat de Barcelona

2 Institut de Recerca Cervell, Cognició i Conducta (IR3C)

\begin{abstract}
The satisfaction of consumers and users with the products and services is an issue that in recent years has been taking more and more importance and now it even reaches psychotherapy. This paper presents the Spanish adaptation of the Consumer Reports Effectiveness Scale (CRES-4) consisting of four items designed to evaluate whether patients are satisfied with the therapy they have received and if it has been perceived as effective or not. Its global score is intended to reflect treatment effectiveness as perceived by the patient. Existing studies suggest that the CRES-4 is a good complementary tool to judge, in particular, satisfaction with treatment received.

Keywords: Client satisfaction, scales adaptation, psychotherapy, perceived improvement after treatment
\end{abstract}

El campo de la evaluación de la psicoterapia ha sido dominado por instrumentos orientados a valorar el nivel de cambio obtenido con respecto a aspectos como los síntomas, el funcionamiento psicosocial, la calidad de vida, aspectos cognitivos o intrapsíquicos, etc. Por otro lado, a nivel general se han ido instaurando procesos de control de calidad y de medida del grado de satisfacción de los clientes y/o usuarios de servicios de todo tipo. Sin embargo, tanto en la práctica clínica cotidiana como en la investigación existe poca tradición de evaluar el grado de satisfacción con el servicio psicoterapéutico recibido.

A finales de la década de los años 70 se empezaron a desarrollar estudios dedicados a la obtención de un método estandarizado para evaluar la satisfacción de los pacientes con el tratamiento recibido. Se observó la necesidad de involucrar al paciente en las evaluaciones de los servicios asistenciales. Se pretendía conocer el grado en que se habían cumplido sus expectativas, la calidad de la prestación obtenida o la existencia de posibles deficiencias, etc., con un método que fuera cuantificable y con cierta validez y fiabilidad. 
Uno de los cuestionarios de satisfacción más usado en servicios asistenciales de todo el mundo es el Client Satisfaction Questionnaire (CSQ) (Larsen, Attkisson, Hargreaves y Nguyen, 1979). Existen 6 versiones: CSQ-3, CSQ-4, CSQ-8, CSQ18A, CSQ-18B y la versión completa de 31 ítems destinada exclusivamente a fines de investigación. La versión estándar es la CSQ-8; se trata de un cuestionario breve formado por 8 ítems con cuatro opciones de respuesta y una puntuación que va desde 8 a 32. La versión original de este cuestionario está en inglés, pero actualmente el CSQ-8 está disponible en más de 25 idiomas, entre ellos el español (Atkinson, 2012).

A nuestro juicio, una desventaja de este cuestionario es que pregunta de muchas formas la misma cuestión (p. ej., después de preguntar el grado de satisfacción, pregunta si se recomendaría a un amigo o si se volvería al programa de tratamiento, caso de necesitarlo). En nuestra experiencia, la pregunta reiterada sobre lo que esencialmente es un mismo aspecto produce tedio y desconexión, lo que suele resultar en un patrón repetitivo de respuestas con poca variabilidad entre ellas. Por otro lado, el sistema CSQ no es de libre acceso. Para poder administrarlo, además de tener un permiso expreso y por escrito del titular de los derechos de autor, se debe pagar por él una tarifa establecida que varía según la versión de la escala que se quiera usar, el idioma en que esté y el número de usos que se necesiten.

\section{El estudio del Consumer Reports: un hito en la medida de la satisfacción en psicoterapia}

Consumer Reports (CR) es una organización estadounidense, independiente y sin ánimo de lucro fundada en 1963 con la finalidad de proteger a los consumidores y trabajar para promover un comercio justo, equitativo y seguro. Lleva a cabo su actividad principal evaluando los productos existentes en el mercado (automóviles, electrodomésticos, compras, salud, etc.) mediante personas expertas y mediante las encuestas que rellenan los mismos consumidores. A partir de los datos obtenidos se informa a todos los consumidores con la publicación de revistas, libros, guías y boletines de los resultados obtenidos. En España existen también organizaciones similares como la Organización de Consumidores y Usuarios (OCU).

En 1995 la revista Consumer Reports realizó un estudio a gran escala en EE.UU. sobre la eficacia de la terapia psicológica en la práctica clínica habitual y la satisfacción con la misma (véase Seligman, 1995). La revista envió en 1994 a 184.000 lectores un cuestionario que incluía preguntas sobre reparación de automóviles y sobre salud mental; esta última sección debía contestarse si en los últimos 3 años se había buscado ayuda (no necesariamente profesional) para problemas psicológicos. Todo el cuestionario fue respondido por 22.000 personas (el 12\%). De los 6.900 que respondieron la sección de salud mental, 2.800 consultaron sólo a amigos, familiares o sacerdotes y los otros 4.100 buscaron más de una fuente de 
ayuda: profesionales de la salud mental (2.900), médicos de familia (1.100) y grupos de autoayuda (1.300). Así pues, sólo un $42 \%$ de los que habían buscado ayuda para sus problemas psicológicos habían consultado a profesionales de la salud mental: psicólogos (37\%), psiquiatras (22\%), trabajadores sociales (14\%), consejeros matrimoniales $(9 \%)$ y otros (18\%; p.ej., enfermeras psiquiátricas). La escala utilizada para la parte de psicoterapia es la que es objeto del presente estudio y se presenta más adelante. Los principales resultados obtenidos fueron los siguientes:

a) La mayoría de los pacientes tratados por profesionales de la salud mental estuvieron satisfechos con la intervención recibida.

b) El $54 \%$ pensaron que la terapia psicológica les había ayudado mucho y el $36 \%$, algo.

c) Funcionaron mejor los tratamientos largos (más de 6 meses) que los breves (6 meses o menos) y cuanto mayor fue la duración del tratamiento, mayor fue la mejora. Los clientes que obtuvieron mejores resultados valorados por ellos mismos en retrospectiva fueron los que estuvieron más de 2 años en tratamiento. Sin embargo, estos pacientes diferían del resto de la muestra en que: a) tendieron a presentar inicialmente problemas más graves y b) estuvieron de acuerdo en seguir una intervención de larga duración.

d) Los profesionales de la salud mental fueron igual de eficaces que los médicos de familia con tratamientos de hasta 6 meses, pero más eficaces cuando el tratamiento fue más largo.

e) La terapia psicológica y la combinación de esta con la farmacológica fueron igual de eficaces (no se consideraron los trastornos psicóticos ni los bipolares).

f) Informaron mejores resultados los pacientes que se preocuparon por elegir bien a su terapeuta y que adoptaron una actitud activa durante la terapia (ser abierto, trabajar entre sesiones).

g) Informaron peores resultados los pacientes cuya elección de terapeuta o duración de la terapia estuvo limitada por su seguro.

h) Ninguna modalidad de terapia psicológica se mostró superior a otra en ninguno de los problemas considerados.

Como comentan Bados y Garcia-Grau (2011), está claro que estos resultados no pueden ser concluyentes dadas las características del estudio y los problemas metodológicos que presenta. Por ejemplo, el porcentaje de respuesta fue muy bajo, la muestra sólo fue representativa de la clase media adulta, muchos pacientes fueron atendidos por problemas subclínicos, no existió grupo control, y los datos estuvieron basados en autoinformes generales y retrospectivos.

En general, se considera la encuesta del Consumer Reports más como un estudio de la satisfacción de los consumidores que de la utilidad clínica de la 
psicoterapia. Nielsen et al. (2004) hallaron que, en comparación a un cuestionario pasado prospectivamente (Outcome Questionnaire-45, OQ-45; Lambert, et al., 1996), la aplicación retrospectiva a los mismos pacientes del cuestionario del Consumer Reports sobrestimó de modo notable el cambio conseguido. Por otro lado, concluyeron que el componente de la satisfacción estaba relacionado y, por lo tanto covariaba, con la duración y la intensidad del tratamiento no siendo así con los componentes de cambio percibido ni de resolución del problema.

En un estudio posterior del Consumer Reports (2004) se compararon los tratamientos principalmente psicológicos y principalmente farmacológicos para la depresión y la ansiedad según las respuestas de 3.079 suscriptores de la revista que habían buscado ayuda al respecto. Las principales conclusiones fueron:

a) Más del $80 \%$ pensaron que el tratamiento recibido les había ayudado.

b) La terapia farmacológica fue de efectos más rápidos que la psicológica. Sin embargo, ésta dio lugar a mejores resultados cuando el tratamiento duró al menos 13 sesiones. La combinación de ambas terapias fue incluso algo más eficaz. No se informa de resultados de seguimiento una vez descontinuado el tratamiento.

c) Con terapia farmacológica no fue fácil encontrar el fármaco adecuado para cada paciente. El $55 \%$ probaron dos o más fármacos y el $10 \%$, cinco o más.

d) Los fármacos antidepresivos provocaron efectos secundarios en un mayor porcentaje de personas ( $40 \%$ ) que el informado en investigaciones controladas (15\%). Un $4050 \%$ tuvieron problemas sexuales, alrededor de un $20 \%$ ganaron peso y un $15-20 \%$ experimentaron somnolencia o desorientación.

e) El tratamiento aplicado por médicos de asistencia primaria fue tan eficaz como el llevado a cabo por especialistas en salud mental en personas con problemas leves, pero los especialistas obtuvieron mejores resultados con casos graves.

f) Informaron mejores resultados los pacientes que adoptaron una actitud activa: investigaron sobre su problema, se preocuparon por elegir bien a su terapeuta, preguntaron al profesional si tenía experiencia en tratar su problema, buscaron que un amigo o familiar viniera a una sesión, mantuvieron un registro de su tratamiento y estado emocional y aplicaron en su vida diaria lo que estaban aprendiendo en el tratamiento.

g) En la última década se ha incrementado de modo muy notable la aplicación de fármacos para tratar la ansiedad y la depresión, y en cambio se ha reducido el número de sesiones del tratamiento psicológico.

\section{La escala de satisfacción con el tratamiento recibido (CRES-4)}

La escala CRES-4 utilizada en el estudio del Consumer Reports (1995) consta de 4 ítems: una pregunta de satisfacción, una pregunta para calificar el grado de 
resolución del problema principal, una pregunta acerca del estado emocional antes de empezar el tratamiento y, por último, una pregunta del estado emocional en el momento de responder al CRES-4 (véase Anexo). A partir de estas 4 preguntas se pueden extraer tres componentes que corresponden a la percepción del cambio del estado emocional, la satisfacción y la solución de problemas.

\section{La adaptación española de la CRES-4}

Se partió de la descripción de la CRES-4 proporcionada por Nielsen et al. (2004). Dos psicólogos realizaron la traducción al español y posteriormente se realizó una traducción inversa. Las discrepancias encontradas fueron discutidas con un doctor en psicología con más de 25 años de experiencia clínica y académica (GF). La versión resultante (véase Anexo) fue distribuida entre una docena de alumnos del master en Terapia Cognitivo Social de la Universitat de Barcelona para recabar sus comentarios con respecto a la aplicabilidad de la escala. Al no encontrase ningún problema, se aplicó a seis pacientes que estaban siendo tratados en el contexto de las prácticas de dicho master. De dicha aplicación tampoco surgió la necesidad de introducir ningún cambio en los ítems. Por tanto, la versión definitiva coincide con la que se muestra en el anexo.

\section{Corrección}

Tal como explican Nielsen et al (2004), se obtienen tres componentes:

1. Satisfacción. Se basa en la primera pregunta y la respuesta puede variar de 0 a 5 . Se multiplica el valor de la respuesta por la constante 20 para poder obtener puntuaciones que vayan de 0 a 100 . Cuanto más alta es la puntuación, mayor es el grado de satisfacción.

2. Solución del problema. Se basa en la segunda pregunta y la respuesta puede variar de 0 a 5 . También aquí se multiplica el valor de la respuesta por la constante 20 para poder obtener puntuaciones que vayan de 0 a 100 . A mayor puntuación, mayor percepción de que se ha resuelto el problema por el que se ha consultado.

3. Percepción del cambio emocional. Se basa en la tercera y cuarta preguntas. Se resta la puntuación de la respuesta obtenida en la pregunta del estado emocional pre-tratamiento de la puntuación post-tratamiento. Para evitar una posible puntuación negativa, se suman 4 puntos al resultado de esta resta, así se obtiene una puntuación que varía entre $0 \mathrm{y}$ 8. A continuación, el resultado se multiplica por 12,5 para obtener una puntuación entre 0 y 100 . Cuanto más alta es la puntuación final mayor es la mejora en el estado emocional. Puntuaciones por debajo de 50 indican empeoramiento (cuanto más bajas, mayor es el deterioro).

Para obtener la puntuación global de la CRES-4 se suman los resultados de los componentes anteriores tal como representa la siguiente fórmula: 
CRES-4 $=(20 \mathrm{x}$ satisfacción $)+(20 \mathrm{x}$ solución del problema $)+[12,5 \times(4+$ estado emocional actual - estado emocional pre-tratamiento)]

Por lo tanto, para interpretar la CRES-4 de forma global se considera una puntuación que está en una escala que va de 0 a 300 puntos. Cuanto mayor es la puntuación total, mayor es la eficacia del tratamiento según el paciente (Nielsen et al., 2004).

\section{Discusión}

En la actualidad, poder evaluar la satisfacción de un paciente una vez ha finalizado el tratamiento se ha convertido en algo primordial. Ya no solo desde el punto de vista del paciente, el cual puede sentir que su voz es escuchada, sino también para el terapeuta y para el centro donde se desarrolla el tratamiento. Es necesario poder disponer de una herramienta estandarizada para poder comparar los tratamientos de diferentes centros. En este sentido, la CRES-4 se presenta como un primer instrumento para responder a esta necesidad en el mundo de la psicoterapia de habla hispana. En futuros estudios se podrá estudiar su validez y fiabilidad psicométricas y presentar datos de muestras de habla hispana. En cualquier caso, debería reservarse para el estudio meramente de la satisfacción puesto que hay que tener en cuenta las limitaciones que suponen las valoraciones retrospectivas de eficacia y/o efectividad. A este efecto, se recomienda el uso de instrumentos que valoren el cambio terapéutico de forma más precisa (y no retrospectiva) como pueden ser el OQ-45 o bien el recientemente disponible en español CORE-OM (Feixas et al, 2012).

\footnotetext{
La satisfacción de consumidores y usuarios con los productos y servicios es un tema que en los últimos años ha ido adquiriendo cada vez más importancia hasta alcanzar también el campo de la psicoterapia. En este trabajo se presenta la adaptación al español de la Escala de Satisfacción con el Tratamiento Recibido (CRES-4). Tiene cuatro items y fue creada para evaluar el grado de satisfacción del cliente con la terapia recibida, el grado en que considera que su problema principal se ha resuelto y el cambio percibido en su estado emocional del pretratamiento al postratamiento. La puntuación global pretende reflejar la eficacia del tratamiento según el paciente (Nielsen et al., 2004). Los estudios realizados hasta la fecha con este instrumento sugieren que la CRES-4 puede ser un buen instrumento complementario, especialmente si se quiere evaluar la satisfacción con la psicoterapia.

Palabras clave: Satisfacción del cliente, adaptación de escalas, psicoterapia, cambio percibido
} 


\section{ANEXO}

\section{ESCALA DE SATISFACCIÓN CON EL TRATAMIENTO RECIBIDO (CRES-4)}

En general, ¿qué tan satisfecho/a está con la forma en que su terapeuta ha tratado el problema por el que consultó?

0. Completamente insatisfecho/a

1. Muy insatisfecho/a

2. Algo insatisfecho/a

3. Bastante satisfecho/a

4. Muy satisfecho/a

5. Completamente satisfecho/a

¿En qué medida le ha ayudado el tratamiento en relación al problema específico que le llevó a consultar?

0 . No estoy seguro/a

1. Hizo que las cosas empeorasen bastante

2. Hizo que las cosas empeorasen un poco

3. No ha habido cambios

4. Hizo que las cosas mejorasen algo

5. Hizo que las cosas mejorasen mucho

¿Cuál era su estado emocional general cuando empezó el tratamiento?

0 . Estaba muy mal, apenas lograba hacer frente a las cosas

1. Estaba bastante mal, la vida me resultaba a menudo muy dura

2. Regular, tenía mis altibajos

3. Estaba bastante bien, no tenía problemas graves

4. Estaba muy bien, me gustaba mucho la vida que llevaba

¿Cuál es su estado emocional general en este momento?

0 . Estoy muy mal, apenas logro hacer frente las cosas

1. Estoy bastante mal, la vida es por lo general muy dura para mí

2. Regular, tengo mis altibajos

3. Estoy bastante bien, no tengo problemas graves

4. Estoy muy bien, me gusta mucho la vida que llevo

(C) Guillem Feixas y Olga Pucurull

\section{Referencias bibliográficas}

Attkisson, C. (2012).The Client Satisfaction Questionnaire (CSQ). Recuperado el 30 de noviembre 2012, desde http://www.csqscales.com/

Bados, A. y Garcia-Grau, E. (2011). La práctica de la psicología clínica: Contexto, proceso y habilidades. Saarbrücken: Editorial Académica Española.

Consumer Reports (1995, Noviembre). Mental health: Does therapy help? Vol. 61, pp. 734-739. 
Consumer Reports (2004, Octubre). Drugs vs. talk therapy. Vol. 69, pp. 22-29.

Feixas, G., Evans, C., Trujillo, A., Saúl, L. A., Botella, L., Corbella, S., González, E., Bados, A., García-Grau, E. y López-González, M. A. (en prensa). La versión española del CORE-OM: Clinical Outcomes in Routine Evaluation-Outcome Measure. Revista de Psicoterapia.

Lambert, M. J., Hansen, N. B., Umphress, V., Lunnen, K., Okiishi, J., Burlingame, G. M., y Reisinger, C. W. (1996). Administration and scoring manual for the OQ-45.2. East Setauket, NY. American Professional Credentialing Services.

Larsen, D., Attkisson, C., Hargreaves, W. y Nguyen, T. (1979). Assessment of client/patient satisfaction: Development of a general scale. Evaluation and Program Planning, 2, 197-207.

Nielsen, S.L., Smart, D., Isakson, R., Worthen, V., Gregersen, A. y Lambert, M. (2004). The Consumer Reports Effectiveness Score: What did consumers report? Journal of Counseling Psychology, 51, 25-37.

Seligman, M. (1995). The effectiveness of psychotherapy: The Consumer Report Study. American Psychologist, $50,965-974$. 